\title{
Multi-Objective Topology Optimization of Heat Conduction and Linear Elastostatic using Weighted Global Criteria Method
}

\author{
Martin Denk ${ }^{1 *}$, Klemens Rother ${ }^{2}$, Kristin Paetzold ${ }^{1}$ \\ ${ }^{1}$ University of Federal Armed Forces Munich, Institute for Product Development \\ 2 University of Applied Science Munich, Institute of Material and Building Research \\ ${ }^{*}$ Corresponding Author: \\ Martin Denk \\ Universität der Bundeswehr München \\ Werner-Heisenberg-Weg 39 \\ 85579 Neubiberg \\ Mail: martin.denk@unibw.de
}

\begin{abstract}
Multi-Objective Topology Optimization is a tool for finding lowweight solutions using a discretized geometry of several objective functions. In this contribution, the coupling of heat conduction and elastostatics is covered using the global criteria method. For the comparison of the different objectives and objective distributions typically the single target optimized solutions are selected as normalization criteria [1], [2]. The use of these optimized results requires additional calculation effort, so that this work uses the objective distribution of full material properties instead. For the normalization, the objective distributions will be standardized, and quantile normalized. The normalization strategies are compared to each other by the number of iterations and the resulting objective value.
\end{abstract}

\author{
Keywords \\ Multi Objective, Topology Optimization, Global Criteria, Heat \\ Conduction, Elasto Static
}




\section{Introduction}

Topology optimization is typically used for suitable design suggestions for objectives like mean compliance, mean temperature or model analysis. Such optimization often leads to organic-like shapes which require flexible manufacturing like additive manufacturing. Depending on the manufacturing process or the material, some adjustments such as the design of support structures, or the change of shape based on criteria like overhang angles and wall thicknesses are necessary [3], [4]. By ignoring such conditions, the geometry might collapse during production due to its own weight. In addition to the geometrical criteria, thermal loads during the production can lead to thermal stress cracks, significant changes in the resulting shape and altering mechanical properties [3]. Therefore, the component must withstand mechanical and thermal loads during production.

The transfer of mechanical loads as well as the dissipation of heat is required in many other applications such as the support and cooling of a battery [5] or a heat exchanger [6]. In most cases, the function of cooling and the transfer of mechanical loads are considered separately [5], [6], so that suboptimal solutions can appear. In a battery for example, the heat exchanger does not cover any static loads and only a small amount of heat is dissipated via the respective bearing [5]. In order to find suitable design proposals, multi-objective optimization could solve such problems [7]-[10]. In our current research, passive cooling including static mechanical loads is used for a multi-objective topology optimization. We cover both objectives as independent systems without any correlation, so that the temperature and displacement should have no effect on each other. This requirement is satisfied if only small displacements and temperature gradients occur. The combination should result in pareto optimal solutions, so that the areas for cooling and load transfer support each other cooperatively.

\section{State of the Art}

The use of topology optimization is applied in many different areas, such as elastostatic [7], heat conduction [8]-[11], fluid mechanics [8] or dynamics [7], [9]. The optimization of several objective functions can be combined using various approaches such as the weighed sum method [7]-[10], neural networks [12] , the global criteria method [7] or the normal constraint method [13]. Therefore different type of physical objectives can be combined like a) the total pressure energy loss with the mean temperature [8], b) the mean compliance with the mean temperature [10], or the mean compliance with the first natural eigenmode [7]. Additionally to the different types of physical objectives manufacturing costs and assembly costs can also be taken into account [4] by defining cost functions depending on the shape of the resulting geometry.

In our approach we combine heat conduction and elastostatic similar to [10]. As primary goal, [10] minimizes the thermo-mechanical loads by optimizing the head conduction, so that the temperature is distributed equally (Small gradient of temperatures). In contrast to [10], we cover heat dissipation and elastostatic independently, so that the heat dissipation and sustaining mechanical load condition are supporting each other cooperatively. Additionally, our approach should rather increase the heat dissipation than reduce the thermo-mechanical loads.

One main challenge comparing different objectives is finding suitable weight factors [7], [13]. Therefore, [7] uses a min-max feature scaling by selecting the maximum and minimum value of the current sensitivity number of each target. Despite to [7], we use statistical properties for the normalization on the objective distribution, so that the different distribution functions can be compared to each other. In [1] and [2], the optima of each single objective are used directly for scaling the objective distributions, which requires additional optimization steps before iterating with the global criteria. In contrast to [1] and [2], we do not require single target optimization for the normalization. To sum our contribution, we want to investigate the 
following two questions. Can we use the global criteria method for covering heat dissipation and elastostatic? Can we find suitable weight factors by using the standard and quantile normalization on the objective distribution with full material properties?

\section{Multi-Objective Topology Optimization with statistical Normalization}

The global criteria method is selected as optimization strategy. If available, the respective optima of the single targets can be used for the global criteria method, so that the distance between the current solution and the reference optima is used as a metric [7]. These reference optima's are typically calculated by single target optimizations [1], [2]. Additionally, the individual distributions of the objectives are quantile and standard normalized, so that different magnitudes of the mean temperature and the mean compliance are covered in the same scale. The objective distribution is built up by the contribution of each element to the objective value. In standard normalization, the standard deviation of the objective distribution is used for scaling, while quantile normalization equalizes the quantiles of several distributions. The individual distribution functions are then combined using the global criteria method.

The following figure shows the multi-target optimization and normalization. In this work, different load cases for heat conduction and elastostatics are first created. Then, the load cases are evaluated using the finite element method. Based on the system response, the individual sensitivities are calculated and normalized using the respective objective function. Then the individual normalized sensitivities are combined using the global criteria method by the corresponding chosen weights. To avoid a checkerboard pattern, a gaussian filter from digital image processing with a standard deviation of 0,5 is selected. Finally, the element stiffness matrices are adjusted using the design variables.

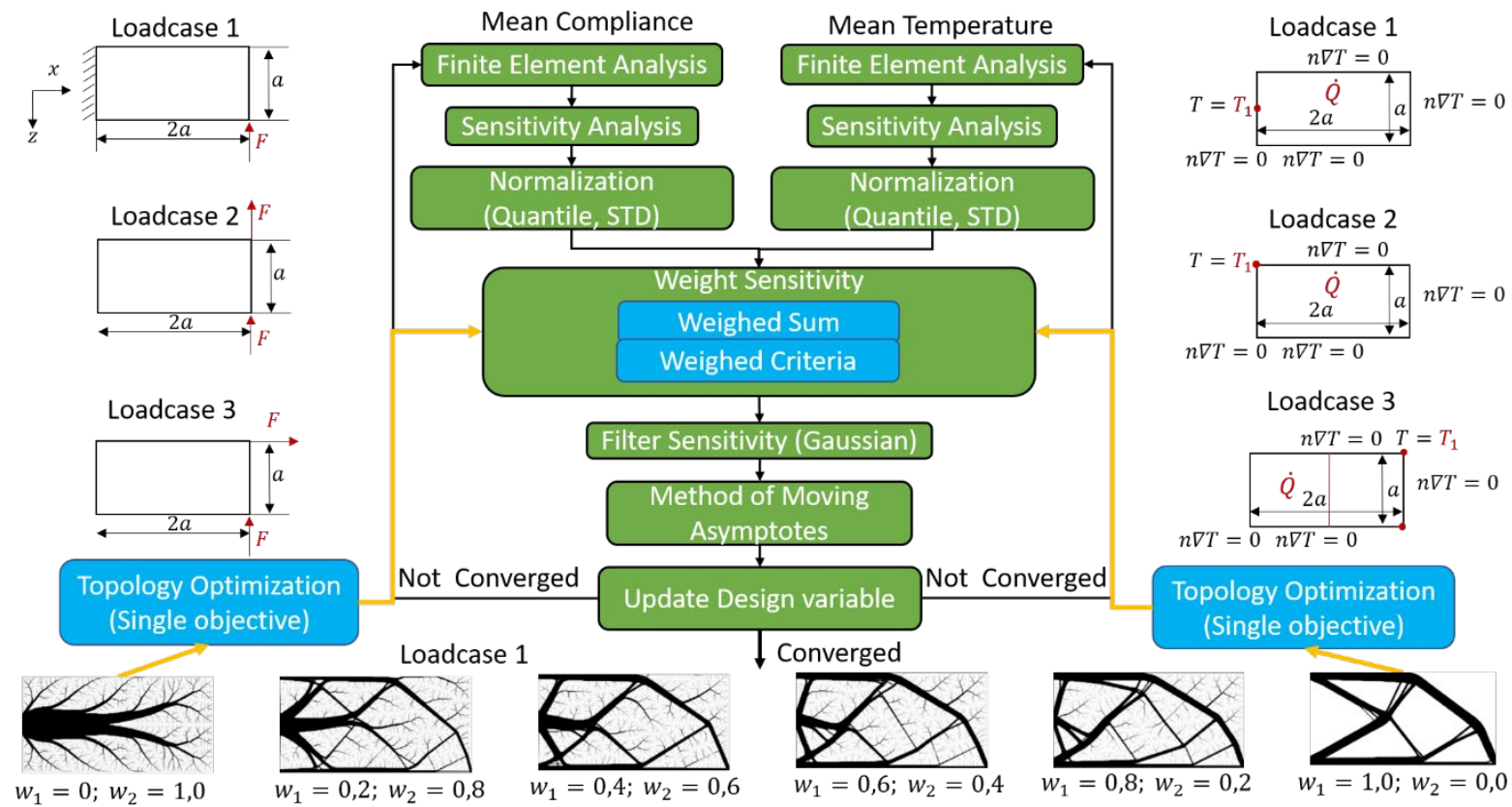

Figure 1: Weighed sum optimization of the two objectives of 1) and 2) with different weight factors using median of quantile normalized distribution

\subsection{Single Objective Optimization}

To obtain the sensitivities, the gradient of the objective function with respect to the design variable must be determined. In topology optimization, the minimization of an objective function such as the mean compliance or mean temperature can be selected with 


$$
\min \left(\Pi=\frac{1}{2} \boldsymbol{x}^{T} \boldsymbol{K} \boldsymbol{x}=\frac{1}{2} \sum_{j=1}^{n} \boldsymbol{x}_{j}^{T} \boldsymbol{k}_{j} \boldsymbol{x}_{j}\right)
$$

where $\boldsymbol{K}$ describes the overall stiffness matrix, $\boldsymbol{x}$ the values of the node degrees of freedom such as displacement values or temperature values, $\boldsymbol{k}_{j}$ the element stiffness matrix and $\boldsymbol{x}_{j}$ the associated displacements on the individual element. So, the value of the objective can be determined by using the overall stiffness matrix or summing up the contributions of each element independently. The objective distribution is a vector which covers the independent contributions of each element with

$$
\boldsymbol{\pi}=\left[\frac{1}{2} \boldsymbol{x}_{1}^{T} \boldsymbol{k}_{1} \boldsymbol{x}_{1} ; \ldots ; \frac{1}{2} \boldsymbol{x}_{n}^{T} \boldsymbol{k}_{n} \boldsymbol{x}_{n}\right]
$$

The design variables $\boldsymbol{\rho}=\left[\rho_{1}, \ldots, \rho_{k}\right]$ are used to change the properties of the stiffness matrix, so that a selected ratio of the volume is sorted in an optimal configuration.

Additionally, the design variables are restricted between 0 and 1 , which represents no, inbetween and full material. To cover these restrictions, the iterative method of moving asymptotes can be chosen. The sensitivity of (1) decides at which areas the material is added and removed. Therefore, equation (1) must be differentiated by using the design variable with

$$
\frac{\partial \Pi}{\partial \rho_{i}}=\frac{1}{2}\left(\frac{\boldsymbol{\partial} \boldsymbol{x}^{T}}{\partial \rho_{i}} \boldsymbol{K} \boldsymbol{x}+\boldsymbol{x}^{T} \frac{\partial \boldsymbol{K}}{\partial \rho_{\boldsymbol{i}}} \boldsymbol{x}+\boldsymbol{x}^{T} \boldsymbol{K} \frac{\partial \boldsymbol{x}}{\partial \rho_{i}}\right)
$$

To find suitable expressions, the different parts of equation (3) can be replaced by the linear system of the finite element analysis and a chosen material interpolation of the material matrix using the design variable. By the differentiation of the linear system $\boldsymbol{K} \boldsymbol{x}=\boldsymbol{f}$ with

$$
\begin{aligned}
& \frac{\partial \boldsymbol{K}}{\partial \rho_{i}} \boldsymbol{x}+\boldsymbol{K} \frac{\partial \boldsymbol{x}}{\partial \rho_{i}}=\frac{\partial \boldsymbol{f}}{\partial \rho_{i}} \\
& \boldsymbol{K} \frac{\partial \boldsymbol{x}}{\partial \rho_{i}}=\frac{\partial \boldsymbol{f}}{\partial \rho_{i}}-\frac{\partial \boldsymbol{K}}{\partial \rho_{i}} \boldsymbol{x}
\end{aligned}
$$

and additionally, the transposition of equation (4) with

$$
\frac{\partial \boldsymbol{x}^{T}}{\partial \rho_{i}} \boldsymbol{K}=\frac{\partial \boldsymbol{f}^{T}}{\partial \rho_{i}}-\boldsymbol{x}^{T} \frac{\partial \boldsymbol{K}}{\partial \rho_{i}}
$$

two expressions of equation (3) can be replaced. By inserting equation (4) and (5) in (3) equation (3) can be reduced to

$$
\begin{gathered}
\frac{\partial \Pi}{\partial \rho_{i}}=\frac{1}{2}\left(\left(\frac{\partial \boldsymbol{f}^{T}}{\partial \rho_{i}}-\boldsymbol{x}^{T} \frac{\partial \boldsymbol{K}}{\partial \rho_{i}}\right) \boldsymbol{x}+\boldsymbol{x}^{T} \frac{\partial \boldsymbol{K}}{\partial \rho_{\boldsymbol{i}}} \boldsymbol{x}+\boldsymbol{x}^{T}\left(\frac{\partial \boldsymbol{f}}{\partial \rho_{i}}-\frac{\partial \boldsymbol{K}}{\partial \rho_{i}} \boldsymbol{x}\right)\right)=\boldsymbol{u} \frac{\partial \boldsymbol{f}^{T}}{\partial \rho_{i}}-\frac{1}{2} \boldsymbol{x}^{T} \frac{\partial \boldsymbol{K}}{\partial \rho_{i}} \boldsymbol{x} \\
\frac{\partial \Pi}{\partial \rho_{i}}=\sum_{j}^{k} u_{j} \frac{\partial f_{j}^{T}}{\partial \rho_{i}}-\frac{1}{2} \sum_{j=1}^{n} \boldsymbol{x}_{j}^{T} \frac{\partial \boldsymbol{k}_{j}}{\partial \rho_{i}} \boldsymbol{x}_{j} .
\end{gathered}
$$

If the design variable should have no influence on the boundary conditions and loads, the equation of (7) can be simplified to 


$$
\frac{\partial \Pi}{\partial \rho_{i}}=-\frac{1}{2} \sum_{j=1}^{n} \boldsymbol{x}_{j}^{T} \frac{\partial \boldsymbol{k}_{j}}{\partial \rho_{i}} \boldsymbol{x}_{j} \text { with } \frac{\partial f_{j}^{T}}{\partial \rho_{i}}=0
$$

Therefore, in equation (8) only one expression is missing for an explicit equation. For the optimization problem, the individual element stiffness matrices $\boldsymbol{k}_{j}$ are adapted using the design variables $\boldsymbol{\rho}$. A function $g\left(\rho_{i}\right)$ can be added to each element stiffness matrix with full material properties $\boldsymbol{k}_{\mathbf{0}}$, so that the resulting element stiffnes matrix is scaled by

$$
\boldsymbol{k}_{j}=g\left(\rho_{j}\right) \boldsymbol{k}_{j}^{0}
$$

Inserting equation (9) in (8) leads to

$$
\frac{\partial \Pi}{\partial \rho_{i}}=\frac{1}{2} \sum_{j=1}^{n} \boldsymbol{x}_{j}^{T} \frac{\partial g\left(\rho_{j}\right)}{\partial \rho_{i}} \boldsymbol{k}_{j}^{0} \boldsymbol{x}_{j}=\frac{\partial g\left(\rho_{i}\right)}{\partial \rho_{i}} \boldsymbol{x}_{i}^{T} \boldsymbol{k}_{i}^{0} \boldsymbol{x}_{i}
$$

Now we can use several different distribution functions depending on the design variable for $g$. If the element stiffness matrix is now explicitly scaled via SIMP model [14] with

$$
g\left(\rho_{i}\right)=\rho_{i}^{u}
$$

the sensitivity can be determined with

$$
\frac{d \Pi}{d \rho_{i}}=-\frac{1}{2} u \rho_{i}^{u-1} \boldsymbol{x}_{i}^{T} \boldsymbol{k}_{i}^{0} \boldsymbol{x}_{i}=-\frac{1}{2} u \rho_{i}^{-1} \boldsymbol{x}_{i}^{T} \boldsymbol{k}_{i} \boldsymbol{x}_{i}
$$

Based on these sensitivity values, the design variables can now be adjusted via the method of moving asymptotes in such a way, that the volume ratio restriction and the range of the design variable can be satisfied, while distributing the material in the proper regions.

\subsection{Multi objective optimization}

Additionally, expression (11) is required for the multi objective criteria. When choosing multi objective criteria, the objective of (1) must be changed. In the following, we combine the load cases by using the global criteria method and a ratio form of the global criteria method. The global criteria method can be defined as a distance metric [1], [2] with

$$
\min \left(\sum_{j=1}^{k} w_{j}\left(\Pi_{j}\right)^{p}\right)^{\frac{1}{p}}
$$

where $w_{j}$ describes the weight and $\Pi_{j}$ the objective of the $j$ th loadcase. The parameter $p$ defines the norm of the metric for the different objectives, so that for the special case $p=1$ the global criteria is reduced to the weighed sum method [7] with

$$
\min \left(\sum_{j=1}^{k} w_{j} \Pi_{j}\right)
$$

Additionally, the global criteria itself can be scaled by using a reference design $\Pi_{j}^{*}$ [7] with the ratio global criteria as 


$$
\min \left(\sum_{j=1}^{k} w_{j}\left(\frac{\Pi_{j}}{\Pi_{j}^{*}}\right)^{p}\right)^{\frac{1}{p}}
$$

(Referenz Design mehr info) For the sensitivity, the differentiation of equation (14) is required. The differentiation of (14) leads to

$$
\frac{\partial \Pi}{\partial \rho_{i}}=\frac{1}{p}\left(\sum_{j=1}^{k} w_{j}\left(\frac{\Pi_{j}(\boldsymbol{\rho})}{\Pi_{j}^{*}}-1\right)^{p}\right)^{\frac{1}{p}-1}\left(\sum_{j=1}^{k} \frac{p w_{j}}{\Pi_{j}^{*}}\left(\frac{\Pi_{j}(\boldsymbol{\rho})}{\Pi_{j}^{*}}-1\right)^{p-1} \frac{\partial \Pi_{j}(\boldsymbol{\rho})}{\partial \rho_{i}}\right) .
$$

With that equation we can now use several approximations of $\Pi_{j}^{*}$. Therefore, we can use several normalization strategies so that the objective $\Pi_{j}(\boldsymbol{\rho})$ is scaled by a reasonable reference design $\Pi_{j}^{*}$.

\subsection{Normalization for the Global Criteria Method}

Finding suitable reference designs $\Pi_{j}^{*}$ in equation (15) or weight factors in equation (12) is not straight forward and leads to additional investigations (optimizations, normalizations, assumptions) [1], [2]. Therefore, we use the contribution of each element to the objective value with full material properties, approximating the reference design. In topology optimization the full material solution covers the optimal solution of the minimization of mean compliance and mean temperature if the ratio of volume is one, so that there is no material distribution. So, for a volume ratio of one we can directly calculate the reference design $\Pi_{j}^{*}$. In our approach we use that reference design as an estimation for the normalization of other volume ratios as well, so that we do not need additional optimizations steps. For the normalization of equation (14), we first use the objective value and the standard deviation of the contribution of each element. Secondly, we use quantile normalization, so that all statistical properties are shared between the objective distribution in $\Pi_{j}^{q, *}$ and $\Pi_{j}^{q, 0}$. Then, we use the standard deviation of the shared distribution for the normalization, so that the normalization depends on the distribution of all objectives.

The following figure covers the different steps of the quantile normalization of [15]. At first, the ordering of the original distributions is stored (Marked with colour). Then, each distribution is sorted according to their value. The distributions are combined to averaging the corresponding sorted values. The resulting shared distribution is used as the reference distribution, so that the averaged values are sorted back to the original positions. The statistical properties of the two distribution functions are now identical. Only the assignment to the individual design variables is different.

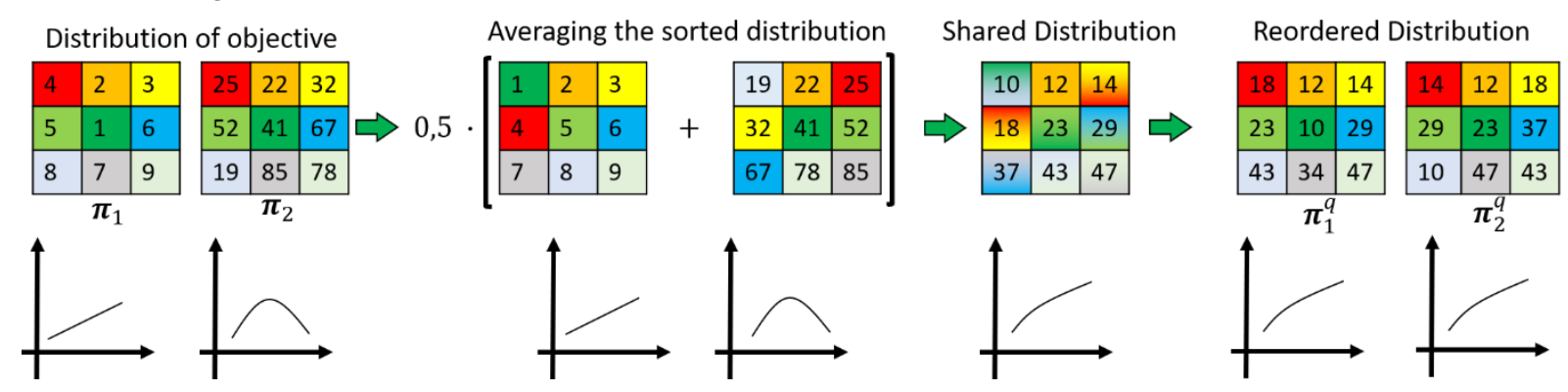

Figure 2: Concept of Quantile Normalisation of an example with different distributions 
Despite the example in figure 2, we first normalize the distribution of each objective with the standard deviation. Otherwise the impact of the shape of the objective distribution with the highest value the shared distribution would be huge, while the influence of other objective distributions could vanish. After the quantile normalization, we use that standard deviation for scaling the distribution back to the origin magnitude. The function in figure 2 shows the alignment of the two distribution functions via a common distribution. This common distribution can now serve for the normalization. The resulting quantile normalized distributions are stored in a vector $\pi_{j}^{q}$ with

$$
\boldsymbol{\pi}_{j}^{q}=\left[\frac{1}{2} \boldsymbol{x}_{1, j}^{q T} \boldsymbol{k}_{1, j}^{q} \boldsymbol{x}_{1, j}^{q} ; \ldots ; \frac{1}{2} \boldsymbol{x}_{n, j}^{q{ }^{T}} \boldsymbol{k}_{n, j}^{q} \boldsymbol{x}_{n, j}^{q}\right]
$$

where $\boldsymbol{x}_{1, j}^{q}{ }^{T} \boldsymbol{k}_{1, j}^{q} \boldsymbol{x}_{1, j}^{q}$ covers the objective value of element 1 of the $j$ th objective. The chosen approximation methods of this contribution are summarized in table 1. Method a) covers the reference case with the optimization of each criteria separately. Method b) uses the objective value with full material properties scaling the corresponding objective. Method c) uses the standard deviation of the distribution of the objective values with full material properties. Method d) uses the standard deviation of the quantile normalized function. Method e) uses the standard deviation of the quantile normalized function of the current objective distribution. Therefore, the weight factors are changing during each iteration dynamically.

Table 1: Weight strategies for the global criteria method

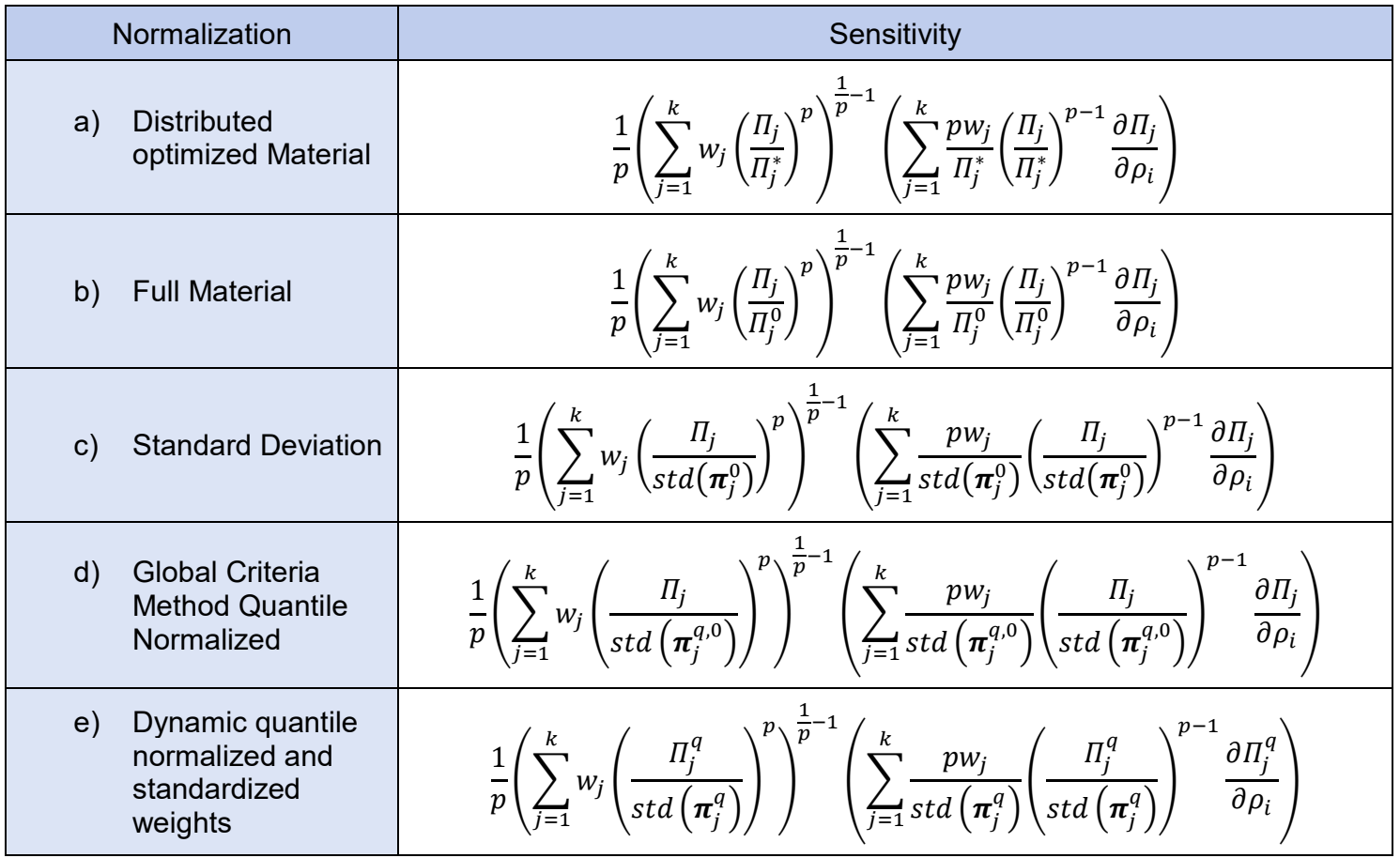

For the comparison of the normalization of table 2 with the global criteria method, we require different metrics. As test criteria we use the weighed sum method summing up the ratios with

$$
\Pi^{g}=w_{1}\left(\frac{\Pi_{1}}{\Pi_{1}^{*}}\right)+w_{2}\left(\frac{\Pi_{2}}{\Pi_{1}^{*}}\right)
$$

and the independent ratios of the single objectives 


$$
\Pi_{1}^{r}=\frac{\Pi_{1}}{\Pi_{1}^{*}} ; \Pi_{2}^{r}=\frac{\Pi_{2}}{\Pi_{2}^{*}}
$$

Equation (17), (18) serves as the reference criteria on the comparison of the different methods. These criteria are used also for exponents larger than one.

\section{Discussion}

The experiment is divided into two scenarios. First, the different exponents of the Global Criteria are compared by using the methods of table 1 and different weight factors by the metric of (16). Second, the most promising methods are used for a visual comparison by choosing different load cases and weight factors. For each use case we calculate the metrices (17), (18) for comparison.

\subsection{Variation of the weight, exponent and method of case 1}

The individual methods from Table 1 are compared using load case 1 under different weights and metric exponents by the criteria of (17). The linear elasto static load case is weighed by $w_{1}$ while the heat conduction case is weighed with $1-w_{1}$. Method a) represents the reference solution that is considered the optimum solution. The methods whose metrics only differ within a tolerance 0,1 from method a) are marked in the table in green.

Table 2: Comparison of the global criteria on different normalization strategies

\begin{tabular}{|c|c|c|c|c|c|c|}
\hline \multirow{2}{*}{ Method } & \multirow{2}{*}{$\begin{array}{c}\text { Weight } \\
w_{1}\end{array}$} & \multicolumn{4}{|c|}{ Weighted Sum (Static) $\Pi^{g}$} & \multirow[t]{2}{*}{ Iterations } \\
\hline & & $p=1$ & $p=2$ & $p=2$ & $p=4$ & \\
\hline \multirow{3}{*}{ a) } & 0,25 & 1,509 & 1,508 & 1,522 & 1,522 & 400 \\
\hline & 0,5 & 1,568 & 1,564 & 1,564 & 1,562 & 400 \\
\hline & 0,75 & 1,522 & 1,526 & 1,532 & 1,535 & 400 \\
\hline \multirow{3}{*}{ b) } & 0,25 & 1,513 & 1,515 & 1,521 & 1,529 & 200 \\
\hline & 0,5 & 1,568 & 1,568 & 1,568 & 1,565 & 200 \\
\hline & 0,75 & 1,523 & 1,525 & 1,530 & 1,534 & 200 \\
\hline \multirow{3}{*}{ c) } & 0,25 & 1,651 & 2,036 & 2,276 & 2,544 & 200 \\
\hline & 0,5 & 1,790 & 1,978 & 2,292 & 2,591 & 200 \\
\hline & 0,75 & 1,635 & 1,934 & 1,987 & 2,039 & 200 \\
\hline \multirow{3}{*}{ d) } & 0,25 & 1,651 & 2,035 & 2,274 & 2,541 & 200 \\
\hline & 0,5 & 1,789 & 1,977 & 2,289 & 2,588 & 200 \\
\hline & 0,75 & 1,635 & 1,934 & 1,986 & 2,038 & 200 \\
\hline \multirow{3}{*}{ e) } & 0,25 & 1,509 & 1,514 & 1,514 & 1,532 & 200 \\
\hline & 0,5 & 1,577 & 1,603 & 1,650 & 144,075 & 200 \\
\hline & 0,75 & 1,595 & 1,659 & 2568,528 & 39,908 & 200 \\
\hline
\end{tabular}

Comparable results to a) can be recorded using methods b) and e) for load case 1 for a metric exponent of one. If the penalty exponent increases to 4 , method $e$ ) is not converging to reasonable values. In the following, method b) and e) will be compared visually and compared by the individual ratios of (18) and the global criterion of (17) using several load cases and various weighting factors. The metric exponent will be set to one. 


\subsection{Variation of the weight factors on case 1 , case 2 and case 3}

In the following, the individual load cases are tested based on the selected normalization b) and e) using further load cases and different weighting factors. For each load case, the global criterion with $p=1$ and the individual ratios of the optima are also given. The lowest values are marked in green, blue, orange for the respective load case 1, 2 and 3 for each weight factor. The results choosing weight factors of $w_{1}=1,0$ and $w_{1}=0,0$ cover the results of the single target optimizations.

Table 3: Variation of the number of elements and load cases with median quantile normalization of weighed sum

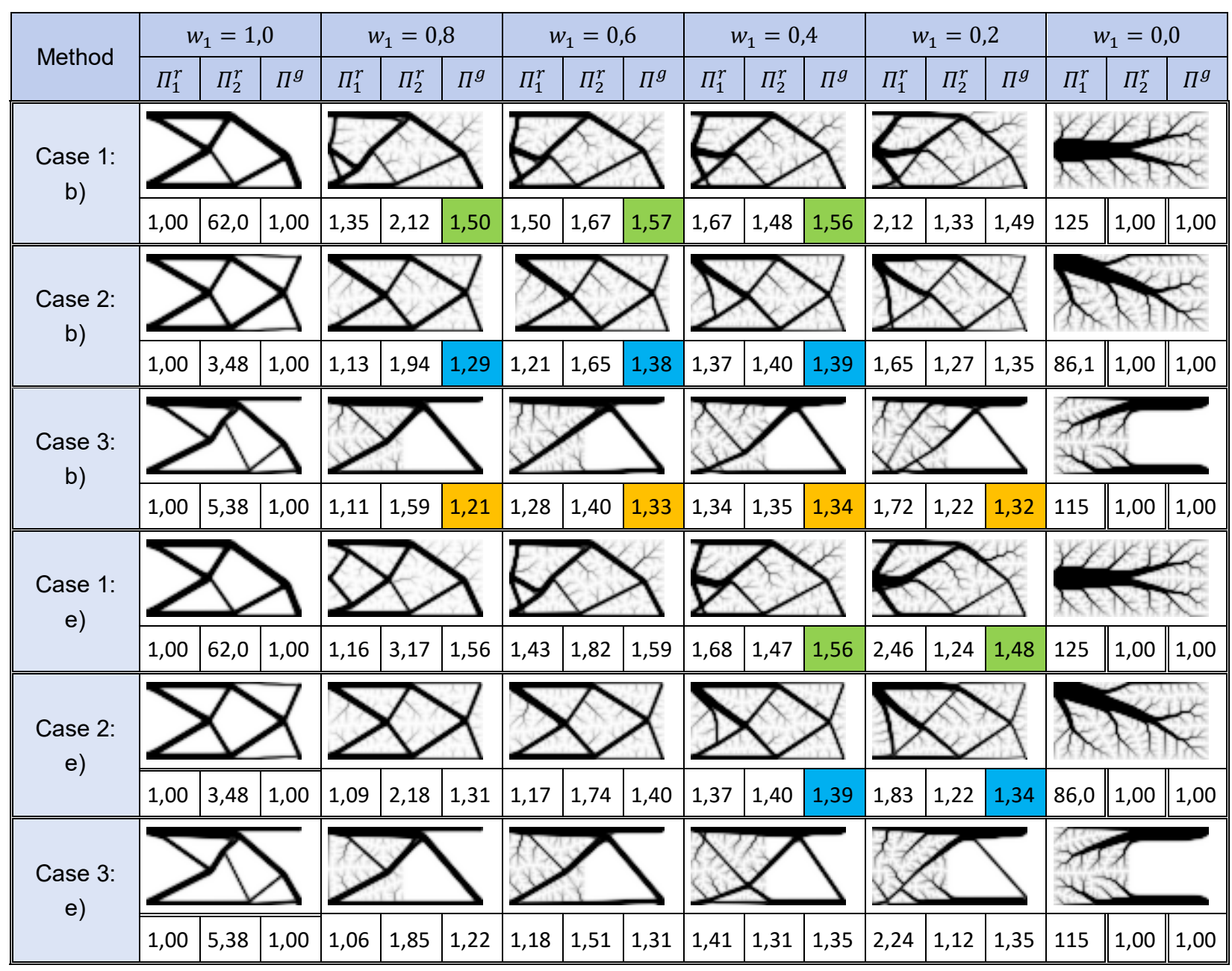

The first evaluation showed that weighting using the objective distribution with full material properties is sufficient and can lower the computation time. The individual static normalization strategies show a suitable utilization, so that a determination of the individual optima is not necessary. Both methods show suitable results for a coupling of heat conduction and elastostatics. Since the magnitudes of the global criterion are similar, both methods can be used. However, because quantile normalization requires additional effort for the normalization, method b) via the sum of the individual contributions of the respective element is more suitable.

So, for further investigations this work can be extended to covering several loadcases for the elastostatic and heat conduction case. Therefore, the global criteria must be adapted, because the elastostatic cases are not independent to each other. Additionally, the objective type can be extended using fluid mechanics [8], natural vibration [7] or cost functions for manufacturing and assembly costs [4]. 


\section{Summary}

The use of multi-target optimization for heat conduction and elastostatics shows cooperately results where for example branches for heat conduction are created at the load-bearing elements. In order to perform a multi-objective optimization using the Global Criteria Method, a normalization strategy is necessary. The normalization allows the individual objective functions to be compared to each other. A suitable normalization can be realized based on the individual optimum of the respective objective function, whereby optimization steps are necessary. To avoid this additional effort, a normalization using a result with full material properties is chosen in this contribution. Based on the resulting objective distribution function, a normalization using the standard deviation and a quantile normalization is applied. The results show similar orders of magnitude as the variant of single optimizations, without additional optimization steps. This approach can be extended in further investigations using manufacturing restrictions and other type of physical objectives.

\section{Literature}

[1] R. V. Grandhi, G. Bharatram, und V. B. Venkayya, „Multiobjective optimization of large-scale structures“, AIAA Journal, Bd. 31, Nr. 7, S. 1329-1337, Juli 1993, doi: 10.2514/3.11771.

[2] P. Hajela und C.-J. Shih, „Multiobjective optimum design in mixed integer and discrete design variable problems", AIAA Journal, Bd. 28, Nr. 4, S. 670-675, Apr. 1990, doi: 10.2514/3.10445.

[3] G. Adam und D. Zimmer, „On design for additive manufacturing: Evaluating geometrical limitations“, Rapid Prototyping Journal, Bd. 21, S. 662-670, 2015, doi: 10.1108/RPJ-06-2013-0060.

[4] D. Guirguis und M. F. Aly, „An evolutionary multi-objective topology optimization framework for welded structures", in 2016 IEEE Congress on Evolutionary Computation (CEC), Juli 2016, S. 372-378, doi: 10.1109/CEC.2016.7743818.

[5] A. Chu, Y. Yuan, J. Zhu, X. Lu, und C. Zhou, „The Design and Investigation of a Cooling System for a High Power Ni-MH Battery Pack in Hybrid Electric Vehicles", Applied Sciences, Bd. 10, S. 1660, 2020, doi: 10.3390/app10051660.

[6] G. Gholamibozanjani und M. Farid, „Experimental and mathematical modeling of an air-PCM heat exchanger operating under static and dynamic loads“, Energy and Buildings, Bd. 202, S. 109354, Nov. 2019, doi: 10.1016/j.enbuild.2019.109354.

[7] K. Proos, G. Steven, O. Querin, und Y. Xie, „Multicriterion evolutionary structural optimization using the weighting and the global criterion methods", AIAA Journal, Bd. 39, S. 2006-2012, 2001, doi: 10.2514/3.14961.

[8] E. Dede, „Multiphysics Topology Optimization of Heat Transfer and Fluid Flow Systems“, 2009.

[9] W.-Y. Kim, R. V. Grandhi, und M. Haney, „Multiobjective Evolutionary Structural Optimization Using Combined Static/Dynamic Control Parameters“, AIAA Journal, Bd. 44, Nr. 4, S. 794-802, 2006, doi: 10.2514/1.16971.

[10] S. Rodríguez und R. Pavanello, „THERMO-MECHANICAL MULTI-OBJECTIVE BIDIRECTIONAL EVOLUTIONARY STRUCTURAL OPTIMIZATION USING WEIGHTED SUM METHOD FOR MEAN COMPLIANCE AND HEAT CONDUCTION PROBLEM“, 2015.

[11] A. Gersborg-Hansen, M. P. Bendsøe, und O. Sigmund, „Topology optimization of heat conduction problems using the finite volume method", Struct Multidisc Optim, Bd. 31, Nr. 4, S. 251-259, Apr. 2006, doi: 10.1007/s00158-005-0584-3.

[12] X. Shao, Z. Chen, M. Fu, und L. Gao, „Multi-objective Topology Optimization of Structures Using NN-OC Algorithms*“, 2007, Bd. 4493, S. 204-212, doi: 10.1007/978-3-540-72395-0_26.

[13] D. J. Munk, T. Kipouros, G. A. Vio, G. T. Parks, und G. P. Steven, „Multiobjective and multi-physics topology optimization using an updated smart normal constraint bi-directional evolutionary structural optimization method“, Struct Multidisc Optim, Bd. 57, Nr. 2, S. 665-688, Feb. 2018, doi: 10.1007/s00158-017-1781-6.

[14] G. Rozvany, "The SIMP method in topology optimization - Theoretical background, advantages and new applications", 2000, doi: 10.2514/6.2000-4738.

[15] B. M. Bolstad, R. A. Irizarry, M. Åstrand, und T. P. Speed, „A comparison of normalization methods for high density oligonucleotide array data based on variance and bias“, Bioinformatics, Bd. 19, Nr. 2, S. 185-193, Jan. 2003, doi: 10.1093/bioinformatics/19.2.185. 